
3 Research Square
Preprints are preliminary reports that have not undergone peer review.
They should not be considered conclusive, used to inform clinical practice, or referenced by the media as validated information.

\title{
Brain 18F-FDG PET for the diagnosis of autoimmune encephalitis: a systematic review and a meta-analysis
}

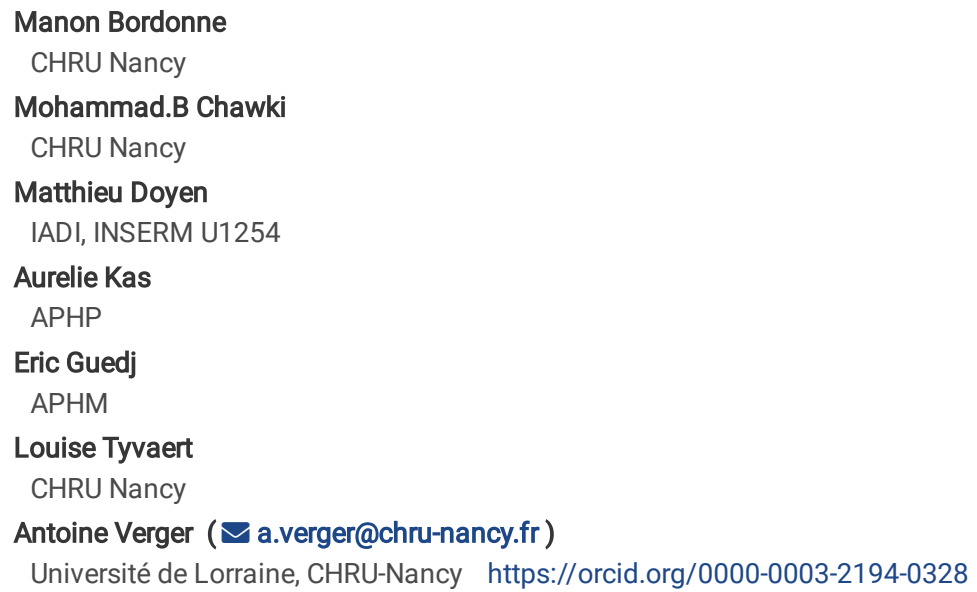

Version of Record: A version of this preprint was published at European Journal of Nuclear Medicine and Molecular Imaging on March 7th, 2021. See the published version at https://doi.org/10.1007/s00259-021-05299-y. 


\section{Abstract}

Objective: To consolidate current understanding of detection sensitivity of brain ${ }^{18} \mathrm{~F}-\mathrm{FDG}$ PET scans in the diagnosis of autoimmune encephalitis and to define specific metabolic imaging patterns for the most frequently occurring autoantibodies.

Methods: A systematic and exhaustive search of data available in the literature was performed by querying the PubMed/MEDLINE and Cochrane databases for the search terms: "FDG PET" and "'encephalitis" or "brain inflammation"'. Studies had to satisfy the following criteria: i. include at least one patient suspected or diagnosed with autoimmune encephalitis according to the current recommendations, ii. be an original case-report iii. specifically present ${ }^{18} \mathrm{~F}$-FDG PET and/or morphologic imaging findings. The diagnostic ${ }^{18}$ F-FDG PET detection sensitivity in autoimmune encephalitis was determined for all cases reported in the literature and a meta-analysis, according to the PRISMA method, was performed on a subset of these, which included PET scans for at least 10 patients, and whose quality was assessed with the QUADAS-2 tool.

Results: The search strategy identified 1113 articles. The detection sensitivity of ${ }^{18} \mathrm{~F}$-FDG PET was $90 \%$, based on 176 publications and 720 patients and $80 \%$ [75\%-84\%] by meta-analysis based on 21 publications and 444 patients. We also report specific brain ${ }^{18} \mathrm{~F}-\mathrm{FDG}$ PET imaging patterns for the main encephalitis autoantibody subtypes.

Conclusion and Relevance: Brain ${ }^{18}$ F-FDG PET has a high detection sensitivity and should be included in future diagnostic autoimmune encephalitis recommendations. Specific metabolic ${ }^{18} \mathrm{~F}$-FDG PET patterns corresponding to the main autoimmune encephalitis autoantibody subtypes further enhance the value of this diagnostic.

\section{Introduction}

Encephalitis is defined as a debilitating neurological disorder that develops as a rapidly progressive encephalopathy caused by brain inflammation ${ }^{1}$. Even though this pathology is relatively rare ${ }^{2}$, its prognosis is poor, entailing serious irreversible sequelae and death in up to $7-12 \%$ of cases ${ }^{3,4}$.

Encephalitis can be subdivided into two main etiologies: infectious (40-52\% of cases ${ }^{5}$ ) and immune mediated (about $21 \%{ }^{4}$ ), which also include the paraneoplastic syndromes, with the remaining cases of unknown origin(s) $6,7,8,9$. Our current study focuses on autoimmune encephalitis, including paraneoplastic encephalitis, associated with onconeuronal antibodies.

Autoimmune encephalitis is characterized by the presence of autoantibodies (aAbs) against neuronal targets ${ }^{10}$. We reviewed encephalitis by aAb subtype, in terms of onconeuronal and non-onconeuronal Abs, since this classification appears to more closely reflect the clinical presentation ${ }^{11}$.

The diagnosis of autoimmune encephalitis is currently based on the clinical and paraclinical criteria defined by Graus et al. in $2016^{1}$. Clinical criteria alone are often inadequate to diagnose autoimmune encephalitis, due to the lack of specificity of symptoms presented by patients ${ }^{12}$. A number of paraclinical tools have therefore been recommended to initially evaluate suspected autoimmune encephalitis cases. These evaluations involve standard biochemistry and immunology tests, to measure the intrathecal synthesis of anti-neuronal-Abs from lumbar punctures. However, it is not uncommon to obtain results several weeks after sampling, moreover, there is no guarantee that these tests will detect any aAbs in CSF at all. This has prompted a search for adjunct diagnostic tools such as EEG and MRI. But EEG results are often non-specific and MRI has a limited $25-50 \%$ sensitivity ${ }^{10,13}$. Because encephalitis patients may suffer severe and sometimes irreversible neurological sequelae, the early initiation of specific treatments and early follow-up of responses to these treatments ${ }^{14}$ are key. This need underscores the importance of finding an early biomarker.

${ }^{18}$ F-Fluoro-deoxy-glucose $\left({ }^{18} \mathrm{~F}-\mathrm{FDG}\right)$ Position Emission Tomography (PET) is a functional brain imaging technique used to visualize neuronal glycolytic metabolic activity which increases during brain inflammation. Importantly, neurological alterations are associated with lower metabolic activity in specific areas of the brain ${ }^{15}$. ${ }^{18} \mathrm{~F}$-FDG PET has also been shown to be superior to morphological imaging in the early diagnosis of autoimmune encephalitis ${ }^{15}$. The increasing number of publications studying the results of brain ${ }^{18} \mathrm{~F}$-FDG PET in autoimmune encephalitis over the past decade is in dire need of consolidation 13-24. The literature is saturated with isolated case-reports and retrospective studies conducted on a small number of patients, leaving the role of PET in the initial assessment of the disease unclear.

Several previous brain ${ }^{18} \mathrm{~F}$-FDG PET in autoimmune encephalitis reviews have attempted to report on the additional benefits of this approach ${ }^{13,15-25}$, but most of these studies focus on the clinical presentation, diagnostic work up, treatments and outcomes even though they all mention ${ }^{18} \mathrm{~F}$-FDG-PET as a useful investigational tool ${ }^{14,16,22,25}$. Conversely, some reviews do focus on distinct ${ }^{18} \mathrm{~F}$-FDG-PET imaging patterns associated of the most commonly detected autoantibodies $13,15,18-21,24$, albeit without providing a systematic or exhaustive review of cases in the literature or a meta-analysis.

Our current systematic and exhaustive literature search completed by a meta-analysis aims to consolidate current understanding of detection sensitivity of

${ }^{18}$ F-FDG PET brain scans in patients diagnosed with autoimmune encephalitis, according to the recommended guidelines (PICOS), and to better define specific metabolic imaging patterns associated with the most commonly occurring autoantibodies.

\section{Materials And Methods}

We queried PubMed/MEDLINE and Cochrane databases, from inception till August 12, 2020, for the search terms: "FDG PET" and "'encephalitis" or "brain inflammation"' to address the specific PICOS question: the diagnostic sensitivity of brain ${ }^{18} \mathrm{~F}$-FDG PET in cortical auto-immune encephalitis. Our systematic and exhaustive search, and meta-analysis, were conducted according to the PRISMA statement ${ }^{26}$.

Page 2/17 
Studies had to satisfy the following criteria: i. include at least one pediatric or adult patient suspected or diagnosed with cortical autoimmune encephalitis (all aAbs including Rasmussen's encephalitis because of its highly probable autoimmune mediation, and aAb associated paraneoplastic syndromes) according to the currently recommended criteria and independently of PET results, ii. be an original case-report (reviews and letters to the editor were excluded), iii. specifically present ${ }^{18} \mathrm{~F}$-FDG PET and/or morphologic imaging findings.

Encephalitis unrelated to cortical autoimmune etiologies (please see Fig. 1 for details) were excluded. All studies satisfying these inclusion criteria were included in the systematic review.

The final selection was performed manually to ensure that all inclusion criteria were satisfied by two independent observers (MB and AV).

We report the number of ${ }^{18}$ F-FDG PET and MRI scans used from each publication, based on one initial diagnosis scan per patient. A true positive scan was defined as specific abnormalities detected in a patient with suspected encephalitis, and the remaining negative scans were considered as false negatives. This allowed the autoimmune encephalitis detection sensitivity to be calculated for both ${ }^{18} \mathrm{~F}$-FDG PET and MRI. To provide a more accurate level of detection sensitivity of encephalitis for MRI and limiting the risk of bias, the analysis was extended to publications with only MRI data to calculate the MRI detection sensitivity. A systematic compilation of ${ }^{18} \mathrm{~F}$-FDG PET detected abnormalities was subsequently determined for each individual aAb. Extractions were repeated, and a final consensus analysis performed (MB and $\mathrm{AV}$ ).

To confirm results of detection sensitivity obtained from our systematic and exhaustive literature search, and to limit the potential bias of overestimating performances linked to the high proportion of published case reports examined, we performed a meta-analysis of ${ }^{18} \mathrm{~F}$-FDG PET performances for the diagnosis of autoimmune encephalitis based on publications analyzing at least 10 patients by PET. The quality of each study was evaluated according to the quality assessment of diagnostic accuracy studies 2 (QUADAS-2) tool by 2 independent investigators (MB and AV); discrepancies were resolved by consensus. Fixed-effect models were performed for the overall analysis and for the aAb analysis. Sensitivities and $95 \%$ confidence intervals are summarized in forest plots. We used the metafor package of R software version 4.0.2 (R Foundation for Statistical Computing, Vienna, Austria). Heterogeneity was evaluated with $\mathrm{I}^{2}$.

\section{Results}

\section{Study design}

The search initially identified 1,113 publications, of which 528 were excluded because they involved non-autoimmune mediated encephalitis. A further 256 publications reporting on non-original cases (reviews, clinical and epidemiologic descriptions) were excluded. 329 full-text publications were finally reviewed and considered for autoimmune encephalitis ${ }^{18}$ F-FDG PET and MRI detection sensitivity. Among these, 176 publications, representing 720 patients, were considered in the PET detection sensitivity calculation, whereas 320 publications (167 publications with both MRI and PET and 153 publications with MRI only, corresponding to 3239 patients) were considered for the MRI detection sensitivity. Among the 176 publications considered in the PET detection sensitivity, 120 were single case reports. For analysis of specific metabolic patterns of the most commonly occurring aAbs, 13 publications were subsequently excluded because they lacked descriptive details of aAb-related anomalies, leaving 163 studies, corresponding to 543 patients, in the analysis.

The study design is summarized as a flowchart (Fig. 1). Supplemental Table 1 details all publications finally included in the analysis of our systematic and exhaustive literature search. The meta-analysis was based on 444 cases from 21 publications ${ }^{27-47}$ each involving at least 10 patients with available brain ${ }^{18}$ F-FDG PET data. QUADAS-2 results are shown in Supplemental Fig. 1.

As detailed in Table 1, brain ${ }^{18} \mathrm{~F}$-FDG PET is associated with an autoimmune encephalitis diagnostic sensitivity of $90 \%$. This compares to an MRI diagnostic sensitivity of $61 \%$ (for MRIs with a corresponding brain ${ }^{18}$ F-FDG PET) and a sensitivity of $56 \%$ when all MRIs were included.

Table 1

Overall performance of PET and MRI detection sensitivity from a systematic and exhaustive search of the autoimmune encephalitis literature

\begin{tabular}{|c|c|c|}
\hline Imaging modality & Number of patients with encephalitis & $\begin{array}{l}\text { Number of patients } \\
\text { with scans suggestive of encephalitis }\end{array}$ \\
\hline${ }^{18} \mathrm{~F}-\mathrm{FDG}$ PET ${ }^{\mathrm{a}}$ & 720 & $645(90 \%)$ \\
\hline MRI in study with $\mathrm{PET}^{\mathrm{a}}$ & 942 & $579(61 \%)$ \\
\hline$M R I^{b}$ & 3,239 & $1,822(56 \%)$ \\
\hline
\end{tabular}

Furthermore, as reported in Fig. 2, forest plots of the meta-analysis showed a detection sensitivity of $80 \%$ [75\%-84\%], with a heterogeneity index $\left(1^{2}\right)$ of $57 \%$. This heterogeneity falls to $21 \%$ when considering specific aAbs.

Brain ${ }^{18}$ F-FDG PET metabolic patterns by specific aAb 


\section{VGKC (Voltage-Gated Potassium Channel) aAbs}

Autoimmune encephalitis involving VGKC aAbs is the form most extensively analyzed by brain ${ }^{18}$ F-FDG PET in the literature ( $n=180$ cases, Table 2 ). These aAbs are directed against cell surface antigens. They can be further classified into: anti-LGI1-Abs (Leucine-rich glioma inactivated 1) and anti-CASPR2-Abs (Contactin-associated protein-like 2) 
Table 2

Summary of encephalitis cases with PET abnormalities by aAb subtype identified from a systematic and exhaustive search of the literature

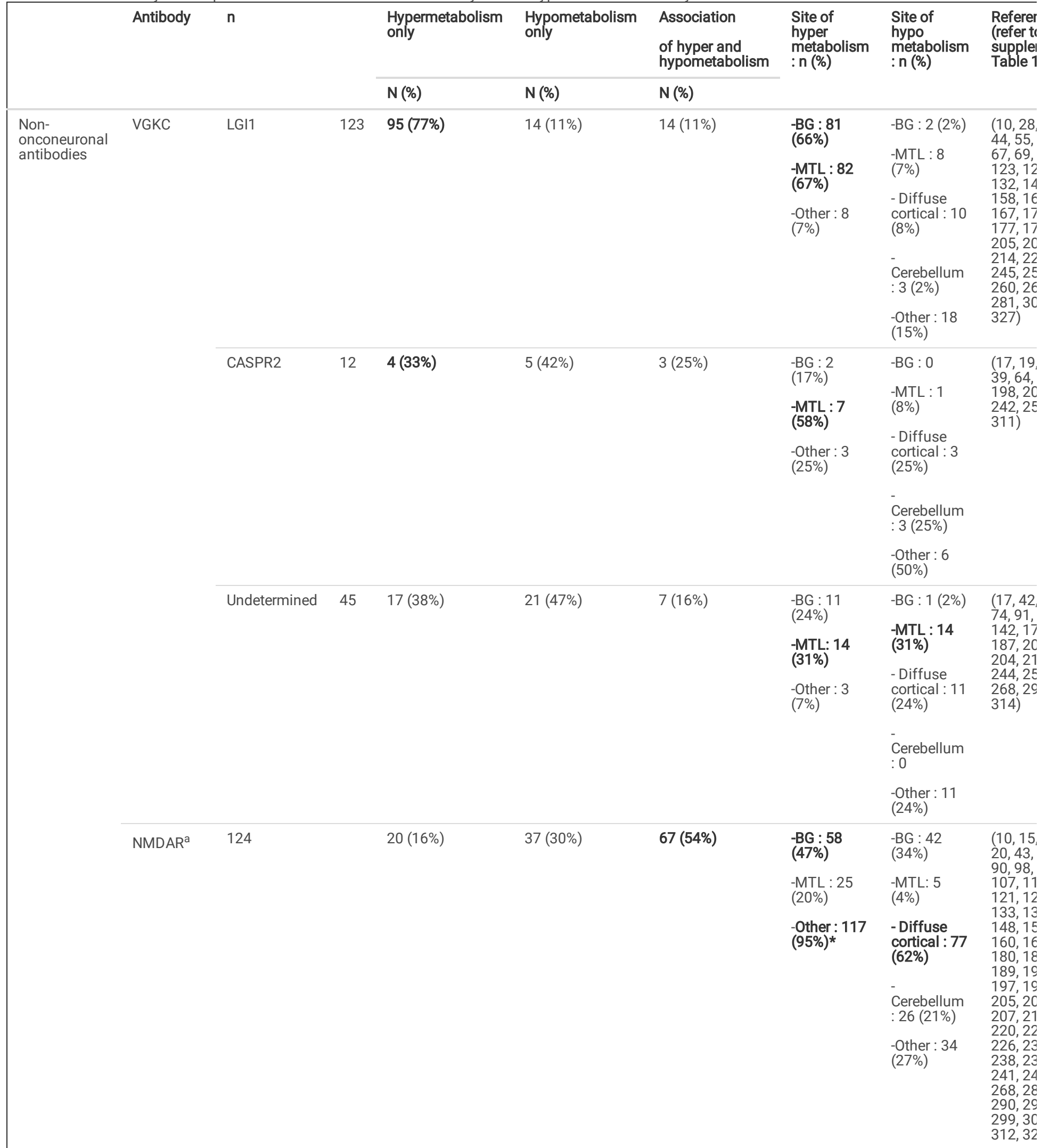

$\mathrm{n}=$ number of PET with abnormal findings. ${ }^{\text {a }}$ hypermetabolism predominant in frontal and temporal lobes associated with hypometabolism in posterior areas hemispheric hypometabolism. ${ }^{c}$ normal findings on visual examination but abnormal metabolism in left MTL on semi-quantitative assessment. ${ }^{d}$ normal finc BG: basal ganglia; MTL: mesial temporal lobe; NMDAR = N-Methyl-D-Asparate Receptor ; VGKC = Voltage-gated potassium channel ; LGI1 = Leucine-rich gliom inactivated 1; CASPR2 = Contactin-associated protein-like $2 ; G A D=$ Glutamic Acid Decarboxylase $; G A B A=$ Gamma-aminobutyric acid $;$ AMPAR = Alpha-Amin Hydroxyl-5-Methyl-4-Isoxazolepropionic acid ; TPO = Thyroid peroxidase ; TG = thyreoglobulin ; DPPX = dipeptidyl-peptidase-like protein 6 ; VGCC = P/Q-type voltage-gated calcium channel; $A C h R=$ anti-acetylcholine receptor; IgLON5 = immunoglobulin-like cell adhesion molecule 5 ; mGluR5 = metabotropic glutama receptor 5 ; IgG4 = immunoglobulin G4. 


\begin{tabular}{|c|c|c|c|c|c|c|c|}
\hline \multirow[t]{2}{*}{ Antibody } & \multirow[t]{2}{*}{$n$} & $\begin{array}{l}\text { Hypermetabolism } \\
\text { only }\end{array}$ & $\begin{array}{l}\text { Hypometabolism } \\
\text { only }\end{array}$ & $\begin{array}{l}\text { Association } \\
\text { of hyper and } \\
\text { hypometabolism }\end{array}$ & \multirow[t]{2}{*}{$\begin{array}{l}\text { Site of } \\
\text { hyper } \\
\text { metabolism } \\
\text { :n (\%) }\end{array}$} & \multirow[t]{2}{*}{$\begin{array}{l}\text { Site of } \\
\text { hypo } \\
\text { metabolism } \\
\text { :n (\%) }\end{array}$} & \multirow[t]{2}{*}{$\begin{array}{l}\text { Refere } \\
\text { (refer } \\
\text { supple } \\
\text { Table }\end{array}$} \\
\hline & & $\mathbf{N}(\%)$ & $\mathbf{N}(\%)$ & $\mathbf{N}(\%)$ & & & \\
\hline GAD & 36 & $8(22 \%)$ & $22(61 \%)$ & $6(17 \%)$ & -BG : 2 (6\%) & -BG : 0 & $\begin{array}{l}(10,17 \\
62,73, \\
268,2 \\
280,2 \\
313)\end{array}$ \\
\hline
\end{tabular}

\begin{tabular}{|c|c|c|c|c|c|c|c|}
\hline & & & & & $\begin{array}{l}\text {-MTL: } 5 \\
(14 \%)\end{array}$ & $\begin{array}{l}-M T L: 22 \\
\text { (61\%) }\end{array}$ & \\
\hline & & & & & $\begin{array}{l}\text {-Other : } 4 \\
(11 \%)\end{array}$ & $\begin{array}{l}\text { - Diffuse } \\
\text { cortical : } 5 \\
(14 \%)\end{array}$ & \\
\hline & & & & & & $\begin{array}{l}\text { Cerebellum } \\
: 1(3 \%)\end{array}$ & \\
\hline & & & & & & $\begin{array}{l}\text {-Other : } 6 \\
(17 \%)\end{array}$ & \\
\hline GABA & 17 & $11(65 \%)$ & $4(24 \%)$ & $2(12 \%)$ & -BG : 2 & -BG : 0 & $(137,1$ \\
\hline & & & & & -MTL : 11 & $\begin{array}{l}-\mathrm{MTL}: 3 \\
(18 \%)\end{array}$ & 277,32 \\
\hline & & & & & $\begin{array}{l}(65 \%) \\
- \text {-Other : } 3 \\
(18 \%)\end{array}$ & $\begin{array}{l}\text { - Diffuse } \\
\text { cortical : } 1 \\
(6 \%)\end{array}$ & \\
\hline & & & & & & $\begin{array}{l}\text { Cerebellum } \\
: 0\end{array}$ & \\
\hline & & & & & & $\begin{array}{l}\text {-Other : } 2 \\
(12 \%)\end{array}$ & \\
\hline AMPAR & 5 & $2(40 \%)$ & $3(60 \%)$ & 0 & $-B G: 0$ & $\begin{array}{l}\text {-BG : } 1 \\
(20 \%)\end{array}$ & $(155,2)$ \\
\hline & & & & & $\begin{array}{l}- \text { MTL : } 1 \\
(20 \%)\end{array}$ & -MTL : 0 & \\
\hline & & & & & $\begin{array}{l}\text {-Other : } 1 \\
(20 \%)\end{array}$ & $\begin{array}{l}\text { - Diffuse } \\
\text { cortical : } 2 \\
(40 \%)\end{array}$ & \\
\hline & & & & & & $\begin{array}{l}\text { Cerebellum } \\
: 0\end{array}$ & \\
\hline & & & & & & $\begin{array}{l}\text {-Other : } 1 \\
(20 \%)\end{array}$ & \\
\hline
\end{tabular}

$\mathrm{n}=$ number of PET with abnormal findings. ${ }^{\text {a }}$ hypermetabolism predominant in frontal and temporal lobes associated with hypometabolism in posterior areas hemispheric hypometabolism. ${ }^{c}$ normal findings on visual examination but abnormal metabolism in left MTL on semi-quantitative assessment. ${ }^{d}$ normal finc BG: basal ganglia; MTL: mesial temporal lobe; NMDAR = N-Methyl-D-Asparate Receptor ; VGKC = Voltage-gated potassium channel ; LGI1 = Leucine-rich gliom inactivated 1; CASPR2 = Contactin-associated protein-like $2 ; G A D=$ Glutamic Acid Decarboxylase ; GABA = Gamma-aminobutyric acid ; AMPAR = Alpha-Amin Hydroxyl-5-Methyl-4-Isoxazolepropionic acid ; TPO = Thyroid peroxidase ; TG = thyreoglobulin ; DPPX = dipeptidyl-peptidase-like protein 6 ; VGCC = P/Q-type voltage-gated calcium channel; $\mathrm{AChR}=$ anti-acetylcholine receptor; IgLON5 = immunoglobulin-like cell adhesion molecule 5 ; mGluR5 = metabotropic glutam: receptor 5 ; IgG4 = immunoglobulin G4. 


\begin{tabular}{|c|c|c|c|c|c|c|c|c|}
\hline \multirow[t]{2}{*}{ Antibody } & \multirow[t]{2}{*}{$n$} & & $\begin{array}{l}\text { Hypermetabolism } \\
\text { only }\end{array}$ & $\begin{array}{l}\text { Hypometabolism } \\
\text { only }\end{array}$ & $\begin{array}{l}\text { Association } \\
\text { of hyper and } \\
\text { hypometabolism }\end{array}$ & \multirow[t]{2}{*}{$\begin{array}{l}\text { Site of } \\
\text { hyper } \\
\text { metabolism } \\
\text { : n (\%) }\end{array}$} & \multirow[t]{2}{*}{$\begin{array}{l}\text { Site of } \\
\text { hypo } \\
\text { metabolism } \\
\text { : } n(\%)\end{array}$} & \multirow[t]{2}{*}{$\begin{array}{l}\text { Referer } \\
\text { (refer t/ } \\
\text { supple } \\
\text { Table 1 }\end{array}$} \\
\hline & & & N (\%) & N (\%) & $\mathbf{N}(\%)$ & & & \\
\hline \multirow[t]{5}{*}{ More rare } & \multirow[t]{5}{*}{ TPO/TG } & \multirow[t]{5}{*}{5} & \multirow[t]{5}{*}{$2(40 \%)$} & \multirow[t]{5}{*}{$3(60 \%)$} & \multirow[t]{5}{*}{0} & $-B G: 0$ & - BG : 0 & \multirow{5}{*}{$\begin{array}{l}(69,75 \\
149,21 \\
228)\end{array}$} \\
\hline & & & & & & $\begin{array}{l}\text {-MTL: } 1 \\
(20 \%)\end{array}$ & -MTL : 0 & \\
\hline & & & & & & & $\begin{array}{l}\text { - Diffuse } \\
\text { cortical : } 2 \\
(40 \%)\end{array}$ & \\
\hline & & & & & & & $\begin{array}{l}- \\
\text { Cerebellum } \\
: 0\end{array}$ & \\
\hline & & & & & & & $\begin{array}{l}\text {-Other : } 1 \\
(20 \%)\end{array}$ & \\
\hline
\end{tabular}

\begin{tabular}{|c|c|c|c|c|c|c|c|}
\hline & & & & & $\begin{array}{l}\text {-Other : } 2 \\
(40 \%)\end{array}$ & & \\
\hline \multirow[t]{6}{*}{ DPPX } & \multirow[t]{6}{*}{2} & \multirow[t]{6}{*}{0} & \multirow[t]{6}{*}{$2(100 \%)$} & \multirow[t]{6}{*}{0} & \multirow{2}{*}{$\begin{array}{l}-B G: 0 \\
-M T L: 0\end{array}$} & \multirow{2}{*}{$\begin{array}{l}- \text { BG : } 1 \\
(50 \%)\end{array}$} & \multirow[t]{6}{*}{$(150,2$} \\
\hline & & & & & & & \\
\hline & & & & & \multirow[t]{4}{*}{-Other : 0} & $(50 \%)$ & \\
\hline & & & & & & $\begin{array}{l}\text { - Diffuse } \\
\text { cortical : } 0\end{array}$ & \\
\hline & & & & & & $\begin{array}{l}- \\
\text { Cerebellum } \\
: 0\end{array}$ & \\
\hline & & & & & & $\begin{array}{l}\text {-Other : } 1 \\
(50 \%)\end{array}$ & \\
\hline \multirow[t]{5}{*}{ VGCC } & \multirow[t]{5}{*}{2} & \multirow[t]{5}{*}{$1\left(50^{\circ} \%\right)$} & \multirow[t]{5}{*}{$1(50 \%)$} & \multirow[t]{5}{*}{0} & $-B G: 0$ & - BG : 0 & \multirow[t]{5}{*}{$(113,3$} \\
\hline & & & & & \multirow{4}{*}{$\begin{array}{l}\text {-MTL : } 1 \\
(50 \%) \\
\text {-Other : } 1 \\
\text { (50\%) }\end{array}$} & -MTL : 0 & \\
\hline & & & & & & $\begin{array}{l}\text { - Diffuse } \\
\text { cortical : } 0\end{array}$ & \\
\hline & & & & & & $\begin{array}{l}\overline{-} \\
\text { Cerebellum } \\
: 0\end{array}$ & \\
\hline & & & & & & $\begin{array}{l}\text {-Other : } 1 \\
(50 \%)\end{array}$ & \\
\hline \multirow[t]{7}{*}{ AChR } & \multirow[t]{7}{*}{2} & \multirow[t]{7}{*}{0} & \multirow[t]{7}{*}{$2(100 \%)$} & \multirow[t]{7}{*}{0} & \multirow{7}{*}{$\begin{array}{l}\text {-BG : } 0 \\
\text {-MTL : } 0 \\
\text {-Other : } 0\end{array}$} & - BG : 0 & \multirow[t]{7}{*}{ (268) } \\
\hline & & & & & & \multirow{2}{*}{$\begin{array}{l}-\mathrm{MTL}: 1 \\
(50 \%)\end{array}$} & \\
\hline & & & & & & & \\
\hline & & & & & & $\begin{array}{l}\text { - Diffuse } \\
\text { cortical : } 1 \\
(50 \%)\end{array}$ & \\
\hline & & & & & & - & \\
\hline & & & & & & $\begin{array}{l}\text { Cerebellum } \\
: 0\end{array}$ & \\
\hline & & & & & & $\begin{array}{l}\text {-Other : } 1 \\
\text { (50\%) }\end{array}$ & \\
\hline
\end{tabular}

$\mathrm{n}=$ number of PET with abnormal findings. ${ }^{\text {a }}$ hypermetabolism predominant in frontal and temporal lobes associated with hypometabolism in posterior areas hemispheric hypometabolism. ${ }^{c}$ normal findings on visual examination but abnormal metabolism in left MTL on semi-quantitative assessment. ${ }^{\mathrm{d}}$ normal finc BG: basal ganglia; MTL: mesial temporal lobe; NMDAR = N-Methyl-D-Asparate Receptor; VGKC = Voltage-gated potassium channel ; LGI1 = Leucine-rich gliom inactivated $1 ;$ CASPR2 = Contactin-associated protein-like $2 ; G A D=$ Glutamic Acid Decarboxylase; GABA = Gamma-aminobutyric acid; AMPAR = Alpha-Amin Hydroxyl-5-Methyl-4-Isoxazolepropionic acid ; TPO = Thyroid peroxidase ; TG = thyreoglobulin ; DPPX = dipeptidyl-peptidase-like protein 6 ; VGCC = P/Q-type voltage-gated calcium channel; $A C h R=$ anti-acetylcholine receptor; IgLON5 = immunoglobulin-like cell adhesion molecule 5 ; mGluR5 = metabotropic glutama receptor 5 ; IgG4 = immunoglobulin G4. 


\begin{tabular}{|c|c|c|c|c|c|c|c|c|}
\hline \multirow[t]{2}{*}{ Antibody } & \multirow[t]{2}{*}{$n$} & & $\begin{array}{l}\text { Hypermetabolism } \\
\text { only }\end{array}$ & $\begin{array}{l}\text { Hypometabolism } \\
\text { only }\end{array}$ & $\begin{array}{l}\text { Association } \\
\text { of hyper and } \\
\text { hypometabolism }\end{array}$ & \multirow[t]{2}{*}{$\begin{array}{l}\text { Site of } \\
\text { hyper } \\
\text { metabolism } \\
: n(\%)\end{array}$} & \multirow[t]{2}{*}{$\begin{array}{l}\text { Site of } \\
\text { hypo } \\
\text { metabolism } \\
\text { : n (\%) }\end{array}$} & \multirow[t]{2}{*}{$\begin{array}{l}\text { Referer } \\
\text { (refer tı } \\
\text { supple } \\
\text { Table 1 }\end{array}$} \\
\hline & & & N (\%) & N (\%) & N (\%) & & & \\
\hline & \multirow{5}{*}{$\begin{array}{l}\text { Amphiphysin } \\
\text { c }\end{array}$} & \multirow[t]{5}{*}{$0^{c}$} & \multirow[t]{5}{*}{0} & \multirow[t]{5}{*}{0} & \multirow[t]{5}{*}{0} & $-B G$ : 0 & $-B G: 0$ & \multirow[t]{5}{*}{ (179) } \\
\hline & & & & & & -MTL : 0 & -MTL : 0 & \\
\hline & & & & & & \multirow[t]{3}{*}{-Other : 0} & $\begin{array}{l}\text { - Diffuse } \\
\text { cortical : } 0\end{array}$ & \\
\hline & & & & & & & $\begin{array}{l}\text { - } \\
\text { Cerebellum } \\
: 0\end{array}$ & \\
\hline & & & & & & & -Other : 0 & \\
\hline & \multirow[t]{5}{*}{ IgLON5 } & \multirow[t]{5}{*}{1} & \multirow[t]{5}{*}{$1(100 \%)$} & \multirow[t]{5}{*}{0} & \multirow[t]{5}{*}{0} & \multirow{5}{*}{$\begin{array}{l}\text {-BG : } 1 \\
(100 \%) \\
\text {-MTL : } 0 \\
\text {-Other : } 1 \\
(100 \%) \star \star \star\end{array}$} & - BG : 0 & \multirow[t]{5}{*}{ (324) } \\
\hline & & & & & & & -MTL : 0 & \\
\hline & & & & & & & $\begin{array}{l}\text { - Diftuse } \\
\text { cortical : } 0\end{array}$ & \\
\hline & & & & & & & $\begin{array}{l}\text { Cerebellum } \\
: 0\end{array}$ & \\
\hline & & & & & & & -Other : 0 & \\
\hline & \multirow[t]{5}{*}{ mGluR5 } & \multirow[t]{5}{*}{3} & \multirow[t]{5}{*}{0} & \multirow[t]{5}{*}{$3(100 \%)$} & \multirow[t]{5}{*}{0} & $-B G: 0$ & - BG : 0 & \multirow[t]{5}{*}{ (271) } \\
\hline & & & & & & -MTL : 0 & -MTL : 0 & \\
\hline & & & & & & -Other : 0 & $\begin{array}{l}\text { - Diffuse } \\
\text { cortical : } 2 \\
(67 \%)\end{array}$ & \\
\hline & & & & & & & $\begin{array}{l}- \\
\text { Cerebellum } \\
: 1(33 \%)\end{array}$ & \\
\hline & & & & & & & -Other : 0 & \\
\hline & \multirow[t]{5}{*}{$\operatorname{lgG} 4$} & \multirow[t]{5}{*}{1} & \multirow[t]{5}{*}{0} & 0 & $1(100 \%)$ & $\begin{array}{l}-B G: 1 \\
(100 \%)\end{array}$ & - BG : 0 & (25) \\
\hline & & & & & & -MTL : 0 & & \\
\hline & & & & & & -Other : 0 & $\begin{array}{l}\text { - Diffuse } \\
\text { cortical : } 0\end{array}$ & \\
\hline & & & & & & & $\begin{array}{l}- \\
\text { Cerebellum } \\
: 0\end{array}$ & \\
\hline & & & & & & & $\begin{array}{l}\text {-Other : } 1 \\
(100 \%)\end{array}$ & \\
\hline & Neuropile & 1 & 0 & 1 & 0 & $-B G$ : 0 & - BG : 0 & (10) \\
\hline & & & & & & -MTL : 0 & -MTL : 0 & \\
\hline & & & & & & -Other : 0 & $\begin{array}{l}\text { - Diffuse } \\
\text { cortical : } 1 \\
(100 \%)\end{array}$ & \\
\hline & & & & & & & $\begin{array}{l}- \\
\text { Cerebellum } \\
: 0\end{array}$ & \\
\hline & & & & & & & -Other : 0 & \\
\hline
\end{tabular}

$\mathrm{n}=$ number of PET with abnormal findings. ${ }^{\text {a }}$ hypermetabolism predominant in frontal and temporal lobes associated with hypometabolism in posterior areas hemispheric hypometabolism. ${ }^{c}$ normal findings on visual examination but abnormal metabolism in left MTL on semi-quantitative assessment. ${ }^{d}$ normal finc BG: basal ganglia; MTL: mesial temporal lobe; NMDAR = N-Methyl-D-Asparate Receptor ; VGKC = Voltage-gated potassium channel ; LGI1 = Leucine-rich gliom inactivated $1 ;$ CASPR2 = Contactin-associated protein-like $2 ; G A D=$ Glutamic Acid Decarboxylase ; GABA = Gamma-aminobutyric acid ; AMPAR = Alpha-Amin Hydroxyl-5-Methyl-4-Isoxazolepropionic acid ; TPO = Thyroid peroxidase ; TG = thyreoglobulin ; DPPX = dipeptidyl-peptidase-like protein 6 ; VGCC = P/Q-type voltage-gated calcium channel; $\mathrm{AChR}=$ anti-acetylcholine receptor; IgLON5 = immunoglobulin-like cell adhesion molecule 5 ; mGluR5 = metabotropic glutam: receptor 5 ; IgG4 = immunoglobulin $\mathrm{G} 4$. 


\begin{tabular}{|c|c|c|c|c|c|c|c|c|}
\hline & \multirow[t]{2}{*}{ Antibody } & \multirow[t]{2}{*}{$n$} & $\begin{array}{l}\text { Hypermetabolism } \\
\text { only }\end{array}$ & $\begin{array}{l}\text { Hypometabolism } \\
\text { only }\end{array}$ & $\begin{array}{l}\text { Association } \\
\text { of hyper and } \\
\text { hypometabolism }\end{array}$ & \multirow[t]{2}{*}{$\begin{array}{l}\text { Site of } \\
\text { hyper } \\
\text { metabolism } \\
: n(\%)\end{array}$} & \multirow[t]{2}{*}{$\begin{array}{l}\text { Site of } \\
\text { hypo } \\
\text { metabolism } \\
\text { : } n(\%)\end{array}$} & \multirow[t]{2}{*}{$\begin{array}{l}\text { Refere } \\
\text { (refer t } \\
\text { supple! } \\
\text { Table } 1\end{array}$} \\
\hline & & & N (\%) & N (\%) & N (\%) & & & \\
\hline \multirow{25}{*}{$\begin{array}{l}\text { Onconeuronal } \\
\text { antibodies }\end{array}$} & \multirow[t]{5}{*}{$\mathrm{Hu}$} & \multirow[t]{5}{*}{11} & \multirow[t]{5}{*}{$6(55 \%)$} & \multirow[t]{5}{*}{3 (27\%) } & \multirow[t]{5}{*}{2 (18\%) } & -BG : 1 (9\%) & -BG : 0 & \multirow{5}{*}{$\begin{array}{l}(17,63 \\
146,17 \\
245,25 \\
268)\end{array}$} \\
\hline & & & & & & $\begin{array}{l}-\mathrm{MTL}: 6 \\
(55 \%)\end{array}$ & $\begin{array}{l}-\mathrm{MTL}: 2 \\
(18 \%)\end{array}$ & \\
\hline & & & & & & $\begin{array}{l}\text {-Other : } 4 \\
(36 \%)\end{array}$ & $\begin{array}{l}\text { - Diffuse } \\
\text { cortical : } 5 \\
(45 \%)\end{array}$ & \\
\hline & & & & & & & $\begin{array}{l}- \\
\text { Cerebellum } \\
: 1(9 \%)\end{array}$ & \\
\hline & & & & & & & -Other : 0 & \\
\hline & \multirow[t]{5}{*}{ Ma $1 / 2$} & \multirow[t]{5}{*}{7} & \multirow[t]{5}{*}{3 (43\%) } & \multirow[t]{5}{*}{$3(43 \%)$} & \multirow[t]{5}{*}{0} & $-B G: 0$ & -BG : 0 & \multirow{5}{*}{$\begin{array}{l}(10,36 \\
216,25 \\
268)\end{array}$} \\
\hline & & & & & & $\begin{array}{l}\text {-MTL: } 2 \\
(29 \%)\end{array}$ & $\begin{array}{l}-\mathrm{MTL}: 3 \\
(43 \%)\end{array}$ & \\
\hline & & & & & & $\begin{array}{l}\text {-Other : } 1 \\
(17 \%)\end{array}$ & $\begin{array}{l}\text { - Diffuse } \\
\text { cortical : } 3 \\
(43 \%)\end{array}$ & \\
\hline & & & & & & & $\begin{array}{l}- \\
\text { Cerebellum } \\
: 0\end{array}$ & \\
\hline & & & & & & & -Other : 0 & \\
\hline & \multirow[t]{5}{*}{ Yo } & \multirow[t]{5}{*}{2} & \multirow[t]{5}{*}{0} & \multirow[t]{5}{*}{$2(100 \%)$} & \multirow[t]{5}{*}{0} & -BG : 0 & $-B G: 0$ & \multirow[t]{5}{*}{$(68,28$} \\
\hline & & & & & & -MTL : 0 & -MTL : 0 & \\
\hline & & & & & & -Other : 0 & $\begin{array}{l}\text { - Diffuse } \\
\text { cortical : } 0\end{array}$ & \\
\hline & & & & & & & $\begin{array}{l}\text { - } \\
\text { Cerebellum } \\
: 2(100 \%)\end{array}$ & \\
\hline & & & & & & & -Other : 0 & \\
\hline & \multirow[t]{5}{*}{ CMRP5 } & \multirow[t]{5}{*}{2} & \multirow[t]{5}{*}{0} & \multirow[t]{5}{*}{$2(100 \%)$} & \multirow[t]{5}{*}{0} & -BG : 0 & $\begin{array}{l}\text {-BG : } 2 \\
(100 \%)\end{array}$ & $(302,3$ \\
\hline & & & & & & $\begin{array}{l}\text {-MTL : } 0 \\
\text {-Other : } 0\end{array}$ & $\begin{array}{l}-\mathrm{MTL}: 1 \\
(50 \%)\end{array}$ & \\
\hline & & & & & & & $\begin{array}{l}\text { - Diffuse } \\
\text { cortical : } 0\end{array}$ & \\
\hline & & & & & & & $\begin{array}{l}- \\
\text { Cerebellum } \\
: 0\end{array}$ & \\
\hline & & & & & & & -Other : 0 & \\
\hline & $C V 2^{d}$ & $0^{d}$ & 0 & 0 & 0 & $-B G: 0$ & -BG :0 & (62) \\
\hline & & & & & & -MTL : 0 & -MTL :0 & \\
\hline & & & & & & -Other : 0 & $\begin{array}{l}\text { - Diffuse } \\
\text { cortical :0 }\end{array}$ & \\
\hline & & & & & & & $\begin{array}{l}- \\
\text { Cerebellum } \\
: 0\end{array}$ & \\
\hline & & & & & & & -Other :0 & \\
\hline
\end{tabular}

$\mathrm{n}=$ number of PET with abnormal findings. ${ }^{\text {a }}$ hypermetabolism predominant in frontal and temporal lobes associated with hypometabolism in posterior areas hemispheric hypometabolism. ${ }^{c}$ normal findings on visual examination but abnormal metabolism in left MTL on semi-quantitative assessment. ${ }^{\mathrm{d}}$ normal finc BG: basal ganglia; MTL: mesial temporal lobe; NMDAR = N-Methyl-D-Asparate Receptor ; VGKC = Voltage-gated potassium channel ; LGI1 = Leucine-rich gliom inactivated 1; CASPR2 = Contactin-associated protein-like $2 ; G A D=$ Glutamic Acid Decarboxylase ; GABA = Gamma-aminobutyric acid ; AMPAR = Alpha-Amin Hydroxyl-5-Methyl-4-Isoxazolepropionic acid ; TPO = Thyroid peroxidase ; TG = thyreoglobulin ; DPPX = dipeptidyl-peptidase-like protein 6 ; VGCC = P/Q-type voltage-gated calcium channel; $\mathrm{AChR}=$ anti-acetylcholine receptor; IgLON5 = immunoglobulin-like cell adhesion molecule 5 ; mGluR5 = metabotropic glutam: receptor 5 ; IgG4 = immunoglobulin G4. 


\begin{tabular}{|c|c|c|c|c|c|c|c|c|}
\hline & \multirow[t]{2}{*}{ Antibody } & \multirow[t]{2}{*}{$\mathbf{n}$} & $\begin{array}{l}\text { Hypermetabolism } \\
\text { only }\end{array}$ & $\begin{array}{l}\text { Hypometabolism } \\
\text { only }\end{array}$ & $\begin{array}{l}\text { Association } \\
\text { of hyper and } \\
\text { hypometabolism }\end{array}$ & \multirow[t]{2}{*}{$\begin{array}{l}\text { Site of } \\
\text { hyper } \\
\text { metabolism } \\
\text { : }(\%)\end{array}$} & \multirow[t]{2}{*}{$\begin{array}{l}\text { Site of } \\
\text { hypo } \\
\text { metabolism } \\
\text { : n (\%) }\end{array}$} & \multirow[t]{2}{*}{$\begin{array}{l}\text { Refere } \\
\text { (refer tt } \\
\text { supple } \\
\text { Table 1 }\end{array}$} \\
\hline & & & $\mathbf{N}(\%)$ & $\mathbf{N}(\%)$ & $\mathbf{N}(\%)$ & & & \\
\hline \multirow{5}{*}{\multicolumn{2}{|c|}{ Ri }} & \multirow[t]{5}{*}{1} & \multirow[t]{5}{*}{0} & \multirow[t]{5}{*}{$1(100 \%)$} & \multirow[t]{5}{*}{0} & $-B G: 0$ & $-B G: 0$ & \multirow[t]{5}{*}{ (17) } \\
\hline & & & & & & \multirow[t]{3}{*}{-MTL : 0} & $\begin{array}{l}\text {-MTL: } 1 \\
(100 \%)\end{array}$ & \\
\hline & & & & & & & $\begin{array}{l}\text { - Diffuse } \\
\text { cortical : } 0 \\
\text { - } \\
\text { Cerebellum } \\
: 0\end{array}$ & \\
\hline & & & & & & & -Other : 0 & \\
\hline & & & & & & -Other: 0 & & \\
\hline \multirow{5}{*}{\multicolumn{2}{|c|}{ Unprecised }} & \multirow[t]{5}{*}{10} & \multirow[t]{5}{*}{$4(40 \%)$} & \multirow[t]{5}{*}{0} & \multirow[t]{5}{*}{$6(60 \%)$} & $-B G: 0$ & $-B G: 0$ & \multirow[t]{5}{*}{$(191,2$} \\
\hline & & & & & & \multirow{4}{*}{$\begin{array}{l}- \text { MTL : } 9 \\
(90 \%) \\
\text {-Other : } 1 \\
(10 \%)\end{array}$} & -MTL :0 & \\
\hline & & & & & & & $\begin{array}{l}\text { - Diffuse } \\
\text { cortical : } 6 \\
(60 \%)\end{array}$ & \\
\hline & & & & & & & $\begin{array}{l}- \\
\text { Cerebellum } \\
: 0\end{array}$ & \\
\hline & & & & & & & -Other :0 & \\
\hline \multirow{5}{*}{\multicolumn{2}{|c|}{ Rasmussen encephalitis ${ }^{\mathbf{b}}$}} & \multirow[t]{5}{*}{36} & \multirow[t]{5}{*}{$8(22 \%)$} & \multirow[t]{5}{*}{$25(69 \%)$} & \multirow[t]{5}{*}{$3(8 \%)$} & $-B G: 2(6 \%)$ & - BG : 5 & \multirow{5}{*}{$\begin{array}{l}(35,37 \\
58,78, \\
102,14 \\
183,22 \\
303,30 \\
307)\end{array}$} \\
\hline & & & & & & $\begin{array}{l}-\mathrm{MTL}: 1 \\
(3 \%)\end{array}$ & -MTL : 0 & \\
\hline & & & & & & $\begin{array}{l}\text {-Other : } 14 \\
(39 \%)\end{array}$ & $\begin{array}{l}\text { - Diffuse } \\
\text { cortical : } 17 \\
(47 \%)\end{array}$ & \\
\hline & & & & & & & $\begin{array}{l}- \\
\text { Cerebellum } \\
: 0\end{array}$ & \\
\hline & & & & & & & $\begin{array}{l}\text {-Other : } 21 \\
(58 \%) * *\end{array}$ & \\
\hline \multirow{5}{*}{\multicolumn{2}{|c|}{ No antibodies }} & \multirow[t]{5}{*}{50} & \multirow[t]{5}{*}{$12(24 \%)$} & \multirow[t]{5}{*}{$22(44 \%)$} & \multirow[t]{5}{*}{$16(39 \%)$} & $\begin{array}{l}-\mathrm{BG}:{ }^{6} \\
(12 \%)\end{array}$ & $\begin{array}{l}-B G: 1 \\
(2 \%)\end{array}$ & \multirow{5}{*}{$\begin{array}{l}(10,11, \\
50,52, \\
62,79, \\
120,13 \\
135,19 \\
205,2 C \\
211,25 \\
257,28 \\
293,29 \\
305)\end{array}$} \\
\hline & & & & & & $\begin{array}{l}-\mathrm{MTL}: 23 \\
(46 \%)\end{array}$ & $\begin{array}{l}-\mathrm{MTL}: 16 \\
(32 \%)\end{array}$ & \\
\hline & & & & & & $\begin{array}{l}\text {-Other : } 13 \\
(26 \%)\end{array}$ & $\begin{array}{l}\text { - Diffuse } \\
\text { cortical : } 16 \\
(32 \%)\end{array}$ & \\
\hline & & & & & & & $\begin{array}{l}\text { Cerebellum } \\
: 2(4 \%)\end{array}$ & \\
\hline & & & & & & & $\begin{array}{l}\text {-Other : } 15 \\
(30 \%)\end{array}$ & \\
\hline
\end{tabular}

$\mathrm{n}=$ number of PET with abnormal findings. ${ }^{\text {a }}$ hypermetabolism predominant in frontal and temporal lobes associated with hypometabolism in posterior areas hemispheric hypometabolism. ${ }^{c}$ normal findings on visual examination but abnormal metabolism in left MTL on semi-quantitative assessment. ${ }^{d}$ normal finc BG: basal ganglia; MTL: mesial temporal lobe; NMDAR = N-Methyl-D-Asparate Receptor ; VGKC = Voltage-gated potassium channel ; LGI1 = Leucine-rich gliom inactivated $1 ;$ CASPR2 = Contactin-associated protein-like $2 ; G A D=$ Glutamic Acid Decarboxylase $; G A B A=$ Gamma-aminobutyric acid $;$ AMPAR = Alpha-Amin Hydroxyl-5-Methyl-4-Isoxazolepropionic acid ; TPO = Thyroid peroxidase; TG = thyreoglobulin ; DPPX = dipeptidyl-peptidase-like protein $6 ; \mathrm{VGCC}=\mathrm{P} / \mathrm{Q}$-type voltage-gated calcium channel; $\mathrm{AChR}=$ anti-acetylcholine receptor ; IgLON5 = immunoglobulin-like cell adhesion molecule 5 ; mGluR5 = metabotropic glutam: receptor 5 ; IgG4 = immunoglobulin G4.

LGI1 associated encephalitis occurs most frequently and typically presents as limbic encephalitis symptoms. It generally affects men in their $40 \mathrm{~s}$ $10,18,20,21,48,49$. This is in contrast to, CASPR2 associated encephalitis which occurs in older men with a greater range of clinical expression predominantly gait 
disorders, neuromyotonia (sometimes associated with Morvan syndrome) and sleep disturbance 10,18,20,21,48,50.

Brain ${ }^{18}$ F-FDG PET imaging is relatively similar with vast regions of hypermetabolism reported for both VGKC encephalitis entities, as detailed in Table 2. The majority of hypermetabolism are nevertheless located within the basal ganglia and mesial temporal lobes when the encephalitis is associated with anti-LGI1Abs. It is interesting that mesial temporal lobe involvement is more frequently described in CASPR2 associated encephalitis. The meta-analysis of anti-VGKCAb mediated encephalitis identified a detection sensitivity of $82 \%$ [56\%-94\%] (supplemental Fig. 2).

\section{NMDAR (N-Methyl-D-Asparate Receptor) aAbs}

NMDAR encephalitis preferentially affects young women and is frequently associated with teratomas. These aAbs specifically bind to the cell surface and are often associated with autoimmune encephalitis resulting in a significant number of cases with brain ${ }^{18} \mathrm{~F}-\mathrm{FDG}$ PET patterns in the literature $(\mathrm{n}=124$, Table 2 ). The clinical presentation usually progresses in four stages. The first is a prodromal phase with unspecific viral-like syndromes. This is followed by a typically psychotic phase. These two phases are usually followed by a mutic phase and finally a hyperkinetic phase with dysautonomia ${ }^{10,18,20,21,48-51}$.

The brain ${ }^{18}$ F-FDG PET patterns are clearly dependent on the phase of the disease with a mix of described hypermetabolism and hypometabolism but with a typical anteroposterior gradient, as detailed in Table 2. The high frequency of hypometabolism, mainly in associative posterior areas, reported in this entity is due to the delayed diagnosis of this encephalitis, the prodromal phase being unspecific. In most of the cases, a mixed pattern of basal ganglia hypermetabolism and diffuse cortical hypometabolism is reported. However, different patterns of hypermetabolism affecting cortical areas other than the basal ganglia and the mesial temporal lobes are also described including a significant proportion of hypometabolic regions in the cerebellum and other cortical areas, which reflects the vast differential clinical expression of this encephalitis. The NMDAR aAb meta-analysis revealed a detection sensitivity of $90 \%$ [75\%-96\%] (supplemental Fig. 2)

\section{GAD (Glutamic Acid Decarboxylase) aAbs}

Encephalitis associated with anti-GAD Abs is a relatively new entity, and thus less-well described in literature. This encephalitis is caused by aAbs directed against synaptic antigens and is principally characterized clinically by Stiff person syndrome and cerebellar ataxia ${ }^{9,10,18,49}$ but also more recently by refractory, mainly temporal, seizures ${ }^{52}$. Consistently with this latter clinical presentation, the 36 cases reported in Table 2 exhibit brain ${ }^{18}$ F-FDG PET patterns mainly involving hypometabolism, associated with delayed diagnoses and typically affecting the mesial temporal lobes. The GAD aAb meta-analysis reported a detection sensitivity of $73 \%$ [55\%-86\%] (supplemental Fig. 2).

\section{GABA (Gamma-AminoButyric Acid) aAbs}

Similarly to GAD associated encephalitis, anti-GABA-Abs also bind to cell membrane antigens. As reported in Table 2,17 cases with brain ${ }^{18} \mathrm{~F}-\mathrm{FDG}$ PET are reported in the literature. In contrast to GAD associated encephalitis, those associated with anti-GABA-Abs lead to more obvious clinical symptoms, including status epilepticus and refractory epilepsy, cognitive deficits, psychiatric symptoms with depression, confusion and mutism ${ }^{10,18,20,21,48,50}$. Once again, the observed brain ${ }^{18} \mathrm{~F}$-FDG PET patterns are related to the clinical characteristics with a majority of hypermetabolism, suggesting obvious early phase symptoms, predominantly involving the mesial temporal lobes. No data are currently available to determine the detection sensitivity of brain ${ }^{18} \mathrm{~F}-\mathrm{FDG}$ PET associated with these aAbs.

\section{AMPAR (Alpha-amino-3-hydroxyl-5-Methyl-4-isoxazolePropionic Acid Receptor) aAbs}

Encephalitis associated with anti-AMPAR-Abs, directed against cell membrane antigens, are poorly described with only 5 cases of brain ${ }^{18} \mathrm{~F}-\mathrm{FDG}$ PET reported in the literature (Table 2). Most of the patients are middle-aged women, with $70 \%$ of cases presenting with diverse tumors. The typical clinical presentation involves a limbic encephalitis, memory impairment, seizures and psychiatric symptoms ${ }^{10,18,20,21,49}$. In line with these diverse clinical manifestations, the observed brain ${ }^{18}$ F-FDG PET patterns are predominantly represented by diverse hypometabolism affecting several cortical areas other than the basal ganglia, the mesial temporal lobes or the cerebellum. Similarly to the anti-GABA-Abs, no publications are available to specifically determine the detection sensitivity of brain ${ }^{18}$ F-FDG PET in this entity.

\section{Onconeuronal aAbs}

Onconeuronal aAbs are found in paraneoplastic encephalitis. Approximately two-thirds of cases involve anti-neuronal-Abs, with neurological symptoms preceding the diagnosis of a tumor by up to 4 years ${ }^{36}$. It is particularly useful to perform whole-body ${ }^{18} \mathrm{~F}$-FDG PET in these entities since the search for a primitive neoplastic tumor as well as dissemination of the primary lesion can be performed at the same time. A typical clinical evolution for this encephalitis subtype remains to be described ${ }^{18,48}$. Brain ${ }^{18}$ F-FDG PET pattern data in the literature are scarce and is based on a total of 30 cases when all anti-neuronalAbs are combined (Table 2). For anti-Hu-Abs, a hypermetabolism of the mesial temporal lobe is preferentially reported whereas an equivalent number of hyper or hypometabolism in the mesial temporal lobes are described for the anti-Ma $1 / 2$ Abs. In cases involving unspecified aAbs, it appears that hypermetabolic and mixed patterns specifically involving the temporal lobe hypermetabolisms and diffuse cortical hypometabolisms predominate. Interestingly, for the anti-YoAbs, the two reported cases showed hypometabolism of the cerebellum. The onconeuronal aAb meta-analysis revealed a detection sensitivity of $75 \%$ [48\%-90\%] (Supplemental Fig. 2).

\section{Rasmussen's encephalitis}


Rasmussen's encephalitis presumably involves an immune-mediated mechanism even though the pathophysiology of this progressive disease remains unknown. Although recent observations do not exclusively relate to a childhood pathology, a progressive epileptic disorder due to chronic unilateral encephalitis are the two core characteristics ${ }^{53}$. Brain ${ }^{18} \mathrm{~F}$-FDG PET is a useful imaging tool in this setting since a typical pattern of hemispheric hypometabolism, exceeding the atrophy visualized in MRI, is observed and can help to diagnose the disease.

Only 1 publication involving at least 10 patients is available for Rasmussen's encephalitis and yields a sensitivity of $100 \%{ }^{31}$.

Additional observations from other rare aAbs encephalitis ( $\leq 5$ cases in the literature) are detailed in Table 2. We also report the number of encephalitic cases with no specific aAbs $(n=50)$. Due to the probable heterogeneity of entities in this subgroup, the related brain ${ }^{18}$ F-FDG PET patterns are also diverse with a predominance of hypometabolism.

The Fig. 3 illustrates the typical brain ${ }^{18}$ F-FDG PET patterns for the main aAbs entities of autoimmune encephalitis.

\section{Discussion}

Results from our systematic and exhaustive literature search confirm the importance of brain ${ }^{18} \mathrm{~F}-\mathrm{FDG}$ PET in the diagnosis of autoimmune encephalitis and measure an overall detection sensitivity performance of $90 \%$ which is consistent with the meta-analysis (80\% [75\%-84\%]). These diagnostic performances seem to be consistent across the main aAbs subtypes (VGKC, NMDAR, GAD and onconeuronal aAbs, supplemental Fig. 2). The diagnostic sensitivity of ${ }^{18} \mathrm{~F}$ FDG PET from our meta-analysis is clearly higher than that typically reported for brain MRIs (between 56 to $61 \%$ in our current study and 25 to $50 \%$ in the literature ${ }^{48,54}$ ), which underscores the importance of systematically deploying this imaging modality in the initial diagnosis of suspected encephalitis cases. This detection sensitivity for assessing autoimmune encephalitis is very helpful, since diagnosis of the disease is currently delayed due to non-specific clinical symptoms and moderate performances of biological and imaging biomarkers ${ }^{1}$. Neurologists, radiologists and nuclear physicians should also be cognizant of the benefits of brain ${ }^{18} \mathrm{~F}-\mathrm{FDG}$ PET in the initial diagnostic assessment of autoimmune encephalitis.

The different brain ${ }^{18} \mathrm{~F}$-FDG PET pattern results from our exhaustive literature search underlines a similarity observed among the neurodegenerative diseases $55,{ }^{18}$ F-FDG PET abnormalities are strongly related to clinical symptoms. This allows typical brain ${ }^{18} \mathrm{~F}$-FDG PET patterns to be defined. The mesial temporal lobe involvement is observed in autoimmune encephalitis associated with limbic encephalitis (VGKC, GAD, GABA aAbs). Autoimmune encephalitis associated with a variety of clinical symptoms yield mixed hyper and hypometabolic patterns (NMDAR aAbs). Hypermetabolic patterns are associated with more obvious clinical symptoms (GABA aAbs) than hypometabolic ones (GAD aAbs), but the delay between the onset of symptoms and ${ }^{18} \mathrm{~F}-\mathrm{FDG}$ PET needs to be taken into account, as hypermetabolism is also observed in the acute phase of disease. In certain specific cases, the brain ${ }^{18} \mathrm{~F}$-FDG PET pattern is quasi pathognomonic of an autoimmune encephalitis entity (Rasmussen's encephalitis) with more extensive metabolic alteration than morphologic anomalies. Moreover, to reveal additional specific signs of encephalitis in paraneoplastic syndromes associated with autoimmune encephalitis, ${ }^{18} \mathrm{~F}$-FDG PET is able to provide an extensive assessment of neoplasia by performing a whole-body ${ }^{18}$ F-FDG PET simultaneously to the brain ${ }^{18}$ F-FDG PET 56,57 .

It would have been interesting to report the specificity and accuracy of brain ${ }^{18} \mathrm{~F}$-FDG PET imaging in autoimmune encephalitis. Indeed, some brain ${ }^{18} \mathrm{~F}$-FDG PET patterns, especially diffuse hypometabolism, are not specific and are also observed in neurodegenerative diseases ${ }^{55}$. Unfortunately, false positive results or true negative cases can only be identified from well-conducted prospective studies which are rare in the current literature ${ }^{27,29}$. The performance and ${ }^{18} \mathrm{~F}$ FDG PET patterns observed in our meta-analysis are mainly influenced by the time from the onset of symptoms, which is not always clearly reported in studies, thereby mistaking patterns of hyper- and hypometabolic areas, which may be related to the course of the disease ${ }^{15,58}$.

Overall, our current systematic and exhaustive literature search and meta-analysis focus on sensitivity diagnostic performances as well as specific brain ${ }^{18} \mathrm{~F}$ FDG PET patterns in autoimmune encephalitis. We report the convincing performance of brain ${ }^{18} \mathrm{~F}-\mathrm{FDG}$ PET in the diagnosis of autoimmune encephalitis, which provides a helpful diagnostic imaging tool to overcome the challenges of diagnosing this entity and underscores the importance of including diagnostic ${ }^{18}$ F-FDG PET in any future recommendations. Specific metabolic patterns corresponding to the main autoimmune encephalitis Ab subtypes value-adds to the diagnostic assessment. Further prospective studies are nevertheless required to further define performances in terms of specificity and accuracy.

\section{Declarations}

\section{Funding}

None

\section{Conflicts of interest/competing interests}

The authors disclose no potential conflicts of interest related to the present work.

\section{Ethics approval and consent to participate}

This article does not contain any studies with human participants or animals performed by any of the authors.

\section{Consent for publication}

The consent has been obtained for each patient for whom their FDG PET images are included in the manuscript. 
The data that support the findings of this study are available on request from the corresponding author (AV)

\section{Code availability}

Not applicable

\section{Authors' contributions}

All authors contributed significantly to the analysis and interpretation of the data (MB, MD, MBC, AV), to the writing of the manuscript (EM, AV) and to the revision of the manuscript (EG, AK, LT, AV).

\section{References}

1. Graus F, Titulaer MJ, Balu R, et al. A clinical approach to diagnosis of autoimmune encephalitis. Lancet Neurol. 2016;15(4):391-404. doi:10.1016/S14744422(15)00401-9

2. Jmor F, Emsley HC, Fischer M, Solomon T, Lewthwaite P. The incidence of acute encephalitis syndrome in Western industrialised and tropical countries. Virol J. 2008;5(1):134. doi:10.1186/1743-422X-5-134

3. Titulaer MJ, McCracken L, Gabilondo I, et al. Treatment and prognostic factors for long-term outcome in patients with anti-NMDA receptor encephalitis: an observational cohort study. The Lancet Neurology. 2013;12(2):157-165. doi:10.1016/S1474-4422(12)70310-1

4. Granerod J, Ambrose HE, Davies NW, et al. Causes of encephalitis and differences in their clinical presentations in England: a multicentre, populationbased prospective study. The Lancet Infectious Diseases. 2010;10(12):835-844. doi:10.1016/S1473-3099(10)70222-X

5. Mailles A, Stahl J. Infectious Encephalitis in France in 2007: A National Prospective Study. CLIN INFECT DIS. 2009;49(12):1838-1847. doi:10.1086/648419

6. Cózar Santiago MDP, Sanchez Jurado R, Sanz Llorens R, Aguilar Barrios JE, Ferrer Rebolleda J. Limbic Encephalitis Diagnosed With 18F-FDG PET/CT: Clinical Nuclear Medicine. 2016;41(2):e101-e103. doi:10.1097/RLU.0000000000001076

7. Lancaster E, Martinez-Hernandez E, Dalmau J. Encephalitis and antibodies to synaptic and neuronal cell surface proteins. Neurology. 2011;77(2):179-189. doi:10.1212/WNL.0b013e318224afde

8. Rosenfeld MR, Dalmau J. Paraneoplastic Neurologic Disorders: A Brief Overview. Memo. 2012;5(3):197-200. doi:10.1007/s12254-012-0034-z

9. McKeon A. Paraneoplastic and Other Autoimmune Disorders of the Central Nervous System. Neurohospitalist. 2013;3(2):53-64. doi:10.1177/1941874412453339

10. Dutra LA, Abrantes F, Toso FF, Pedroso JL, Barsottini OGP, Hoftberger R. Autoimmune encephalitis: a review of diagnosis and treatment. Arq Neuropsiquiatr. 2018;76(1):41-49. doi:10.1590/0004-282X20170176

11. Darnell RB, Posner JB. Paraneoplastic Syndromes Involving the Nervous System. N Engl J Med. 2003;349(16):1543-1554. doi:10.1056/NEJMra023009

12. Armangue T, Leypoldt F, Dalmau J. Autoimmune encephalitis as differential diagnosis of infectious encephalitis: Current Opinion in Neurology. 2014;27(3):361-368. doi:10.1097/WC0.0000000000000087

13. Heine J, Prüss H, Bartsch T, Ploner CJ, Paul F, Finke C. Imaging of autoimmune encephalitis-Relevance for clinical practice and hippocampal function. Neuroscience. 2015;309:68-83. doi:10.1016/j.neuroscience.2015.05.037

14. Diaz-Arias LA, Pardo CA, Probasco JC. Autoimmune Encephalitis in the Intensive Care Unit. In: Nelson SE, Nyquist PA, eds. Neurointensive Care Unit. Current Clinical Neurology. Springer International Publishing; 2020:249-263. doi:10.1007/978-3-030-36548-6_17

15. Guerin J, Watson RE, Carr CM, Liebo GB, Kotsenas AL. Autoimmune epilepsy: findings on MRI and FDG-PET. Br J Radiol. 2019;92(1093). doi:10.1259/bjr.20170869

16. Kyritsis AP, Markoula S, Alexiou G, et al. Diagnosis and treatment of limbic encephalitis in the cancer patient. Future Oncol. Published online June 8, 2020. doi:10.2217/fon-2020-0080

17. Wang H, Xiao Z. Current Progress on Assessing the Prognosis for Anti-N-Methyl-D-Aspartate Receptor (NMDAR) Encephalitis. Biomed Res Int. 2020;2020:7506590. doi:10.1155/2020/7506590

18. Husari KS, Dubey D. Autoimmune Epilepsy. Neurotherapeutics. 2019;16(3):685-702. doi:10.1007/s13311-019-00750-3

19. Bacchi S, Franke K, Wewegama D, Needham E, Patel S, Menon D. Magnetic resonance imaging and positron emission tomography in anti-NMDA receptor encephalitis: A systematic review. Journal of Clinical Neuroscience. 2018;52:54-59. doi:10.1016/j.jocn.2018.03.026

20. Dalmau J, Geis C, Graus F. Autoantibodies to Synaptic Receptors and Neuronal Cell Surface Proteins in Autoimmune Diseases of the Central Nervous System. Physiological Reviews. 2017;97(2):839-887. doi:10.1152/physrev.00010.2016

21. Arbizu J, Giuliani A, Gállego Perez-Larraya J, et al. Emerging clinical issues and multivariate analyses in PET investigations. Q J Nucl Med Mol Imaging. 2017;61(4):386-404. doi:10.23736/S1824-4785.17.03024-2

22. Venkatraman A, Opal P. Paraneoplastic cerebellar degeneration with anti-Yo antibodies - a review. Ann Clin Trans/ Neurol. 2016;3(8):655-663. doi:10.1002/acn3.328

23. Lim M, Gorman M. Autoimmune neurologic disorders in children. Handb Clin Neurol. 2016;133:485-510. doi:10.1016/B978-0-444-63432-0.00026-8

24. Quartuccio N, Caobelli F, Evangelista L, et al. The role of PET/CT in the evaluation of patients affected by limbic encephalitis: A systematic review of the literature. J Neuroimmunol. 2015;284:44-48. doi:10.1016/j.jneuroim.2015.05.002

Page $13 / 17$ 
25. Lee SK, Lee S-T. The Laboratory Diagnosis of Autoimmune Encephalitis. Published online 2016:8.

26. Moher D, Liberati A, Tetzlaff J, Altman DG, PRISMA Group. Preferred reporting items for systematic reviews and meta-analyses: the PRISMA statement. J Clin Epidemiol. 2009;62(10):1006-1012. doi:10.1016/j.jclinepi.2009.06.005

27. Kerik-Rotenberg N, Diaz-Meneses I, Hernandez-Ramirez R, et al. A Metabolic Brain Pattern Associated With Anti-N-Methyl-D-Aspartate Receptor Encephalitis. Psychosomatics. 2020;61(1):39-48. doi:10.1016/j.psym.2019.08.007

28. Turpin S, Martineau P, Levasseur M-A, et al. 18F-Flurodeoxyglucose positron emission tomography with computed tomography (FDG PET/CT) findings in children with encephalitis and comparison to conventional imaging. Eur J Nucl Med Mol Imaging. 2019;46(6):1309-1324. doi:10.1007/s00259-01904302-x

29. Schubert J, Brämer D, Huttner HB, et al. Management and prognostic markers in patients with autoimmune encephalitis requiring ICU treatment. Neurol Neuroimmunol Neuroinflamm. 2018;6(1). doi:10.1212/NXI.0000000000000514

30. Probasco JC, Solnes L, Nalluri A, et al. Abnormal brain metabolism on FDG-PET/CT is a common early finding in autoimmune encephalitis. Neurol Neuroimmunol Neuroinflamm. 2017;4(4):e352. doi:10.1212/NXI.0000000000000352

31. Fiorella DJ, Provenzale JM, Coleman RE, Crain BJ, Al-Sugair AA. (18)F-fluorodeoxyglucose positron emission tomography and MR imaging findings in Rasmussen encephalitis. AJNR Am J Neuroradiol. 2001;22(7):1291-1299.

32. Malter MP, Helmstaedter C, Urbach H, Vincent A, Bien CG. Antibodies to glutamic acid decarboxylase define a form of limbic encephalitis. Ann Neurol. 2010;67(4):470-478. doi:10.1002/ana.21917

33. Aupy J, Collongues N, Blanc F, Tranchant C, Hirsch E, De Seze J. Encéphalites dysimmunitaires, données cliniques, radiologiques et immunologiques. Revue Neurologique. 2013;169(2):142-153. doi:10.1016/j.neurol.2012.05.014

34. Baumgartner A, Rauer S, Mader I, Meyer PT. Cerebral FDG-PET and MRI findings in autoimmune limbic encephalitis: correlation with autoantibody types. Journal of Neurology. 2013;260(11):2744-2753. doi:10.1007/s00415-013-7048-2

35. Shin Y-W, Lee S-T, Shin J-W, et al. VGKC-complex/LGI1-antibody encephalitis: Clinical manifestations and response to immunotherapy. Journal of Neuroimmunology. 2013;265(1-2):75-81. doi:10.1016/j.jneuroim.2013.10.005

36. Masangkay N, Basu S, Moghbel M, Kwee T, Alavi A. Brain 18F-FDG-PET characteristics in patients with paraneoplastic neurological syndrome and its correlation with clinical and MRI findings. Nucl Med Commun. 2014;35(10):1038-1046. doi:10.1097/MNM.0000000000000163

37. Wegner F, Wilke F, Raab P, et al. Anti-leucine rich glioma inactivated 1 protein and anti-N-methyl-D-aspartate receptor encephalitis show distinct patterns of brain glucose metabolism in 18F-fluoro-2-deoxy-d-glucose positron emission tomography. BMC Neurol. 2014;14(1):136. doi:10.1186/1471-2377-14-136

38. Flanagan EP, Kotsenas AL, Britton JW, et al. Basal ganglia T1 hyperintensity in LGI1-autoantibody faciobrachial dystonic seizures. Neurol Neuroimmunol Neuroinflamm. 2015;2(6). doi:10.1212/NXI.0000000000000161

39. Solnes LB, Jones KM, Rowe SP, et al. Diagnostic Value of ${ }^{18}$ F-FDG PET/CT Versus MRI in the Setting of Antibody-Specific Autoimmune Encephalitis. $J$ Nucl Med. 2017;58(8):1307-1313. doi:10.2967/jnumed.116.184333

40. Jang Y, Lee S-T, Bae J-Y, et al. LGI1 expression and human brain asymmetry: insights from patients with LGI1-antibody encephalitis. J Neuroinflammation. 2018;15. doi:10.1186/s12974-018-1314-2

41. Steriade C, Moosa ANV, Hantus S, Prayson RA, Alexopoulos A, Rae-Grant A. Electroclinical features of seizures associated with autoimmune encephalitis. Seizure. 2018;60:198-204. doi:10.1016/j.seizure.2018.06.021

42. Tripathi M, Tripathi M, Roy SG, et al. Metabolic topography of autoimmune non-paraneoplastic encephalitis. Neuroradiology. 2018;60(2):189-198. doi:10.1007/s00234-017-1956-2

43. Falip M, Rodriguez-Bel L, Castañer S, et al. Hippocampus and Insula Are Targets in Epileptic Patients With Glutamic Acid Decarboxylase Antibodies. Front Neurol. 2018;9:1143. doi:10.3389/fneur.2018.01143

44. Lv R-J, Pan J, Zhou G, et al. Semi-quantitative FDG-PET Analysis Increases the Sensitivity Compared With Visual Analysis in the Diagnosis of Autoimmune Encephalitis. Front Neurol. 2019;10:576. doi:10.3389/fneur.2019.00576

45. Strohm T, Steriade C, Wu G, Hantus S, Rae-Grant A, Larvie M. FDG-PET and MRI in the Evolution of New-Onset Refractory Status Epilepticus. American Journal of Neuroradiology. 2019;40(2):238-244. doi:10.3174/ajnr.A5929

46. Deuschl C, Rüber T, Ernst L, et al. 18F-FDG-PET/MRI in the diagnostic work-up of limbic encephalitis. Treglia G, ed. PLoS ONE. 2020;15(1):e0227906. doi:10.1371/journal.pone.0227906

47. Liu X, Shan W, Zhao X, et al. The Clinical Value of 18F-FDG-PET in Autoimmune Encephalitis Associated With LGI1 Antibody. Front Neurol. 2020;11:418. doi:10.3389/fneur.2020.00418

48. Venkatesan A, Adatia K. Anti-NMDA-Receptor Encephalitis: From Bench to Clinic. ACS Chem Neurosci. 2017;8(12):2586-2595. doi:10.1021/acschemneuro.7b00319

49. Leypoldt F, Armangue T, Dalmau J. Autoimmune encephalopathies: Autoimmune encephalopathies. Ann NY Acad Sci. 2015;1338(1):94-114. doi:10.1111/nyas. 12553

50. Najjar S, Steiner J, Najjar A, Bechter K. A clinical approach to new-onset psychosis associated with immune dysregulation: the concept of autoimmune psychosis. J Neuroinflammation. 2018;15. doi:10.1186/s12974-018-1067-y

51. Staley EM, Jamy R, Phan AQ, Figge DA, Pham HP. N-Methyl- d -aspartate Receptor Antibody Encephalitis: A Concise Review of the Disorder, Diagnosis, and Management. ACS Chem Neurosci. 2019;10(1):132-142. doi:10.1021/acschemneuro.8b00304

52. Goudot M, Frismand S, Hopes L, et al. GAD65-Ab encephalitis and subtle focal status epilepticus. Epileptic Disord. 2019;21(5):437-442. doi:10.1684/epd.2019.1094 
53. Varadkar S, Bien CG, Kruse CA, et al. Rasmussen's encephalitis: clinical features, pathobiology, and treatment advances. Lancet Neurol. 2014;13(2):195205. doi:10.1016/S1474-4422(13)70260-6

54. Dalmau J, Lancaster E, Martinez-Hernandez E, Rosenfeld MR, Balice-Gordon R. Clinical experience and laboratory investigations in patients with antiNMDAR encephalitis. The Lancet Neurology. 2011;10(1):63-74. doi:10.1016/S1474-4422(10)70253-2

55. Nobili F, Arbizu J, Bouwman F, et al. European Association of Nuclear Medicine and European Academy of Neurology recommendations for the use of brain 18 F-fluorodeoxyglucose positron emission tomography in neurodegenerative cognitive impairment and dementia: Delphi consensus. Eur J Neurol. 2018;25(10):1201-1217. doi:10.1111/ene.13728

56. Hansen N, Widman G, Stuff S, et al. Cancer frequency detected by positron emission tomography-computed tomography in limbic encephalitis. Epilepsy Behav. 2018;89:105-111. doi:10.1016/j.yebeh.2018.09.043

57. Kampe KKW, Rotermund R, Tienken M, et al. Diagnostic Value of Positron Emission Tomography Combined with Computed Tomography for Evaluating Critically III Neurological Patients. Front Neurol. 2017;8. doi:10.3389/fneur.2017.00033

58. Leypoldt F, Buchert R, Kleiter I, et al. Fluorodeoxyglucose positron emission tomography in anti-N-methyl-D-aspartate receptor encephalitis: distinct pattern of disease. J Neurol Neurosurg Psychiatry. 2012;83(7):681-686. doi:10.1136/jnnp-2011-301969

\section{Figures}

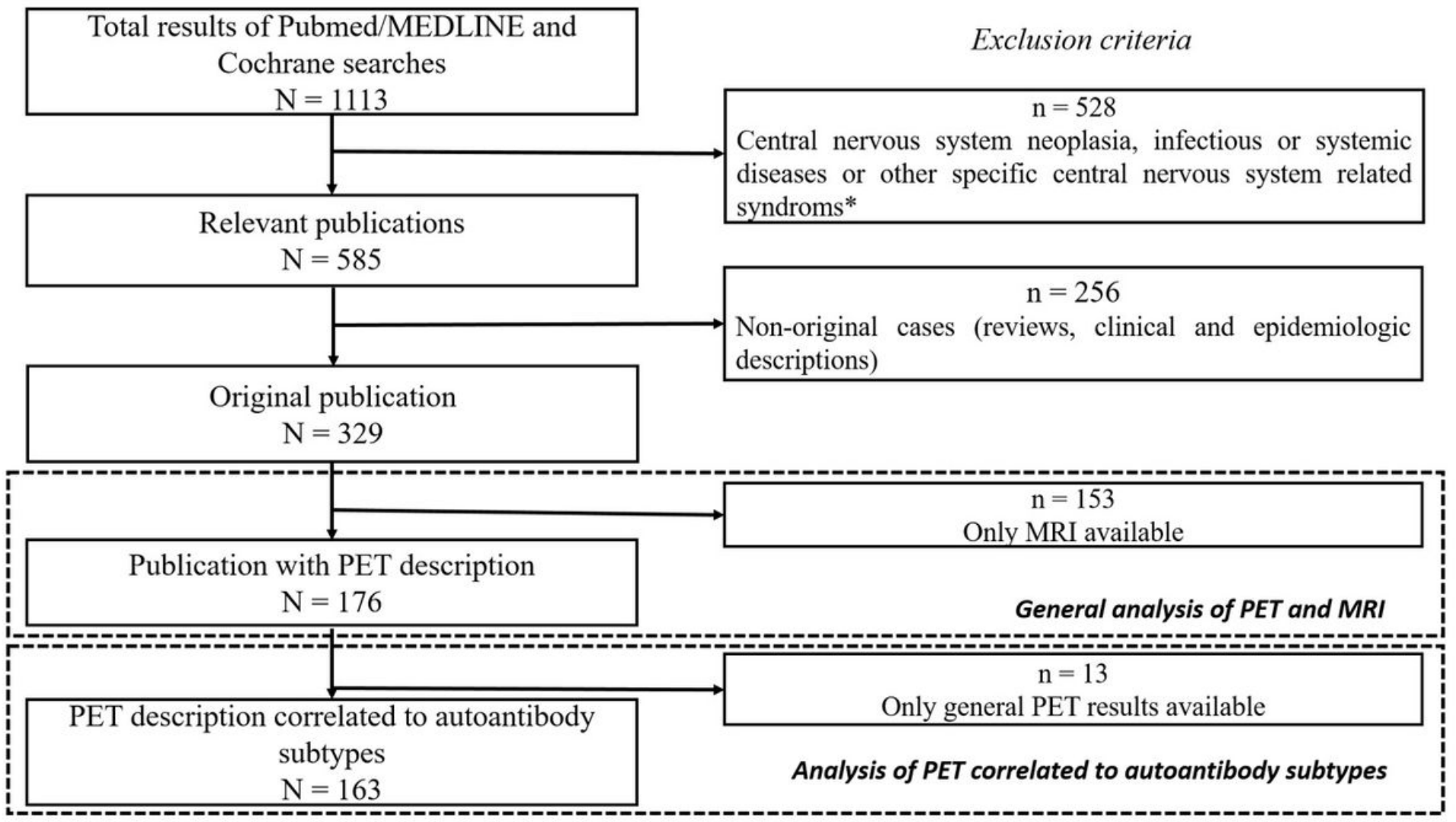

Figure 1

Flowchart of publication selection. * Bickerstaff's brainstem encephalitis, Stiff Person syndrome, CLIPPERS : Chronic Lymphocytic Inflammation with Pontine Perivascular Enhancement Responsive to Steroids, paraneoplasic cerebellar degeneration, subacute sclerosing encephalitis (Measles encephalitis), neurolupus, sarcoidosis, Creutzfeldt-Jakob disease, Morvan syndrome, PML : Progressive multifocal encephalopathy, PANDAS : Pediatric Autoimmune Neuropsychiatric Disorders Associated with Streptococcal Infections, demyelinating syndromes such as ADEM : Acute disseminated encephalomyelitis and PERM : Progressive encephalomyelitis with rigidity and myoclonus 


\section{Meta-analysis}

Ficrela 2n01

Mater 2010

Aupy 2013

Baumgatnen 2013

Stin 2013

Miagangkay 2014

Wegner 2014

Flanagan 2015

Probasco 2017

Sohes 2017

Jang 2018

Ftenade 2018

Falip 2019

Sv 2019

Sctubert 2019

Stroum 2019

Deuschl 2020

Kerik-Rotenberg 2020

Liv 2020

FE Mode

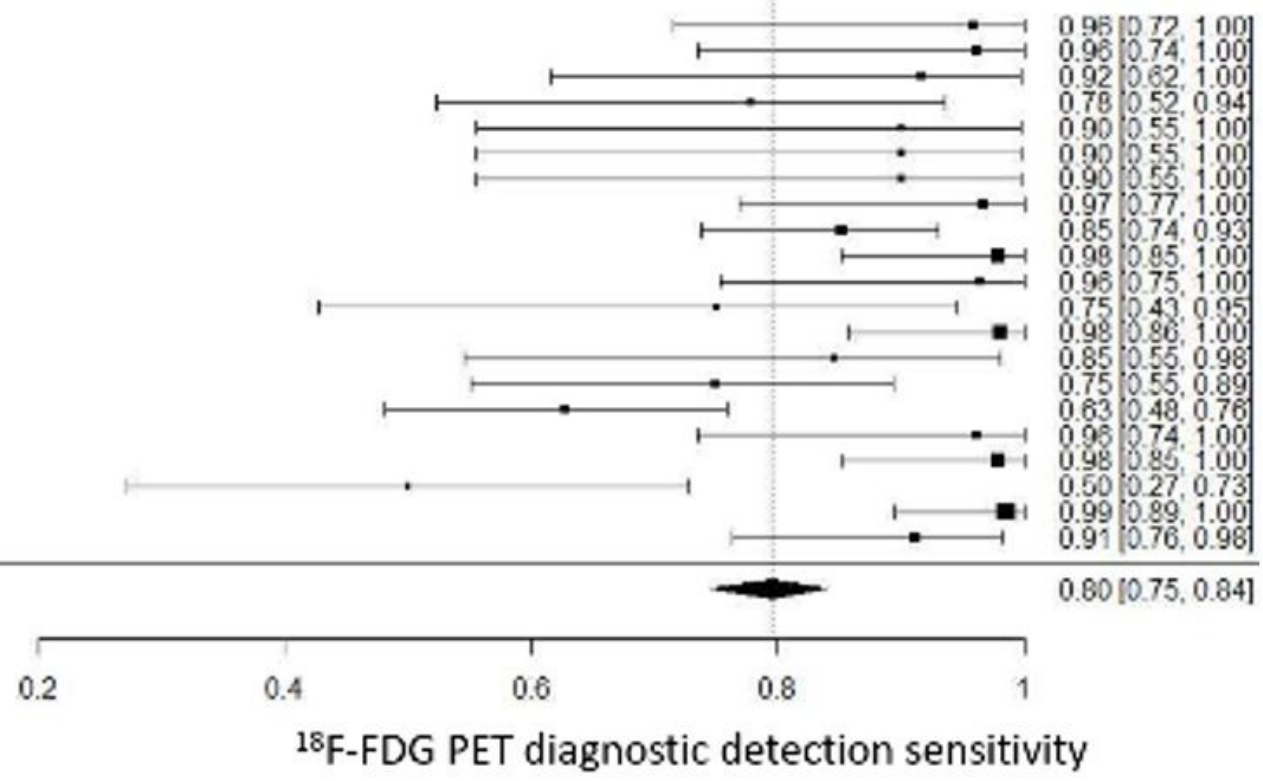

Figure 2

Forest plots of the meta-analysis for the detection sensitivity of brain 18F-FDG PET.

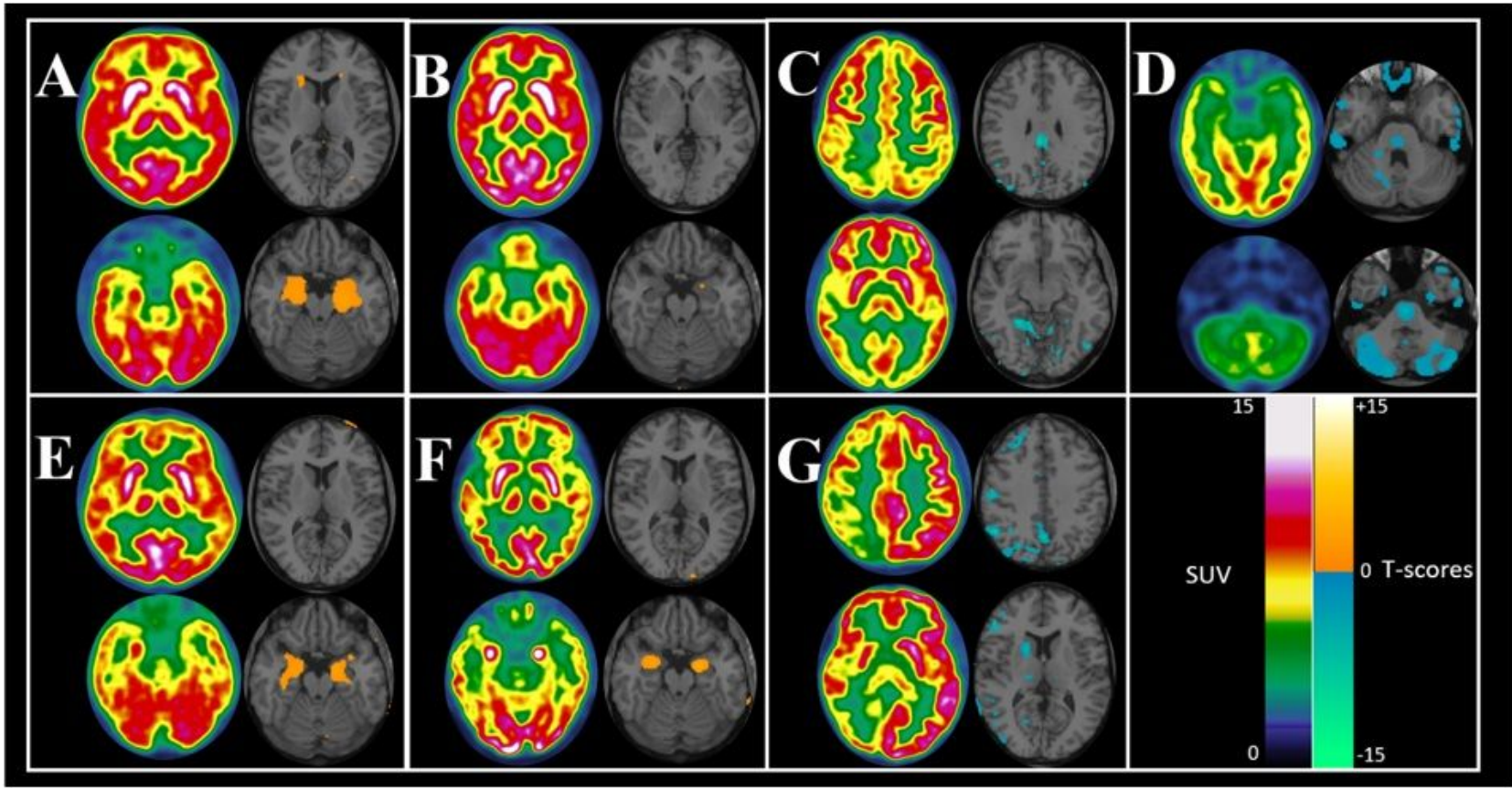

Figure 3 
Image gallery of axial brain 18F-FDG-PET representative of the typical brain 18F-FDG PET patterns for the main aAb entities of autoimmune encephalitis (with the French scale), and corresponding results of Statistical Parametric Mapping (SPM) analyses after comparison to normal controls ( $\mathrm{p}$-voxel<0.005) fused on axial MRI images (hot metal and winter scales). (A) Male patient diagnosed with LGI1 encephalitis. 18F-FDG-PET shows hypermetabolism of bilateral basal ganglia and mesial temporal lobes (MTL). (B) Female patient diagnosed with CASPR2 encephalitis. 18F-FDG-PET shows increased metabolism of the left mesial temporal lobe. (C) Female patient diagnosed with NMDAR encephalitis. The 18F-FDG-PET shows a typical antero-posterior gradient pattern with marqued hypometabolism in the posterior areas and preserved metabolism in cortical anterior areas. (D) Female patient diagnosed with a GAD encephalitis. The typical 18F-FDG-PET pattern shows a temporal hypometabolism involving the mesial part of the lobe. (E) Male patient diagnosed with GABA-B encephalitis. 18F-FDG-PET shows hypermetabolism in bilateral mesial temporal lobes. (F) Female patient diagnosed with autoimmune encephalitis related to anti-Hu antibodies. Typical brain 18F-FDG-PET pattern showed increased metabolism in mesial temporal lobes. (G) Female patient, presenting a Rasmussen encephalitis. 18F-FDG-PET of the brain showed asymmetric metabolism with hypometabolism of right hemisphere and right basal ganglia.

\section{Supplementary Files}

This is a list of supplementary files associated with this preprint. Click to download.

- SupplementalMaterials.pdf

- PRISMA2009ChecklistMSWord.doc

- PRISMA2009FlowDiagramMSWord1.doc 